\title{
Entstehn eines Schweinehinterfulses mit fünf Zehen und der Begleiterscheinungen.
}

\author{
Von \\ Gustar Tornier \\ (Berlin). \\ Mit 13 Figuren im Text. \\ Eingegangen am 12. Juni 1902. \\ Grundriss der Arbeit,
}

Herkunft des Objekts. Statistik der Hyperdaktylie. Kern des Befunds.

Kap. I. Äußeres Bild der Verbildung.

Kap. II. Knocheneharaktere.

1. Astragalus.

2. Naviculare.

3. Tarsale 1 und Entstehn des Überzähligen.

Kap. III. Grund der Tarsale ${ }_{1}$-Zersprengung.

Kap. IV. Indirekte Folgen der Amnionbeanspruchung der Fußwurzel.

Kap. V. Die Muskeln des Stiefzehs.

Kap. VI. Direkter Amnioneinfluss auf den Stiefzeh.

1. Natur des Stiefzehs und seine Amnionbeziehungen.

2. Anpassung des Stiefzehs an die Zwangslage.
a. Metatarsus.
b. Unterer Metatarsuskopf und Gelenk . $_{1}$
c. Gelenk 2 .
d. Gelenk 3 .

Kap. VII. Direkter Amnioneinfluss auf die Stammzehen.

Kap. VIII. Schlussfolgerungen.

1. Verlauf von Knochen- und Gelenkumbildungen.

2. Hant, Muskeln und Knochen unter gemeinsamer technischer Beanspruchung.

3. Über die Daner phylogenetischen und pathologischen Entstehns.

Kap. IX. Außere Antriebe beim Entstehn der Hyperdaktylie.

\section{Vorbemerkung.}

Das Objekt, welches an dieser Stelle besprochen werden soll, ist ein rechter Schweinehinterfuß, welcher an der Innenseite durch 
Tarsale $_{1}$-Zersprengung mit einem Stiefzeh versehen wurde. Ich verdanke die Möglichkeit; ihn und zahlreiche andere ebenso interessante Objekte zu untersuchen, der Giite und Aufopferungsfähigkeit meines wissenschaftlichen Freundes Oberthierarzt Dr. Max Temper in Chemnitz. Herr Dr. Tempel, welcher - als Direktor des Vieh- und Schlachthofs jener Stadt - an einer für die Erwerbung solcher Missbildungen sehr günstigen Stelle sitzt, hatte urspriinglich selbst die Absicht, iiber Polydaktylie zu arbeiten und hatte zu dem Zweek bereits Material und sehr fleiBig Litteratur gesammelt. Da aber inzwischen meine Arbeiten iiber die Entstehungsursachen der Polydaktylie erschienen, gab er nach persönlicher Rücksprache in größter Selbstlosigkeit seinen Plan zu meinen Gunsten anf; und nachdem wir uns alsdann darüber geeinigt hatten, in welcher Weise weiteres Material zu sammeln sei, hat er sich dieser Aufgabe mit unermüdlicher Sorgfalt unterzogen. Beweis dafür ist sein interessanter statistischer Nachweis, dass unter 68248 Schweinen, die in einem Jahre von ihm auf Polydaktylie untersucht wurden, 24, d. h. 0,04\%, damit behaftet waren, während von 16022 Rindern, 27606 Kälbern, 31996 Schafen und 61 Ziegen kein Exemplar derartig verbildet war ${ }^{11}$. So liat er eine Sammlung zusammengebracht, die wohl einzig in ihrer Irt genannt werden kann, und sein Verdienst an den Resultaten dieser Publikation und nachfolgender ist daher ebenso groß wie das meinige.

Als Hauptergebnis meiner Untersuchungen an dem vorliegenden Objekt ergiebt sich nun eine volle Bestätigung der von mir aus friiheren Befunden geschlossenen Thatsache, dass bei Süugethieren das Skelet hyperdaktyler Gebilde ans Knochenwunden entsteht, die in den betreffenden Knochen durch abnorme Amnioneinwirkung erzeugt werden und zwar beansprucht dabei gewöhnlich das Amnion, welches vorher abnorm verlagert ward, einzelne der Gliedmaßenknochen, indem es mit einer Falte auf sie driuckt, so iibermäßig stark anf Biegung, dass diese Knochen im Scheitel der Verbiegung entweder eine Risswunde erhalten oder gar völlig durchbrochen werden. Aus solchen Wunden wachsen dann durch Auslösung von Regenerativkräften die überzähligen Theile der entsprechenden Gliedmaße hervor. In anderen Fällen dringt die Amnionfalte direkt in einen Gliedmaßentheil ein und aus der so entstandenen Wunde wachsen

1) Texres, Zum Vorkommen der Polydaktylie bei unseren schlachtbaren Hausthieren. Zeitschr. f. Fleisch- u. Milchhygiene. 1899. pag. 232. 
dann die überzähligen Gliedmaßentheile hervor. In noch anderen Fällen - wie an dem vorliegenden Fuß - wirkt die Amnionfalte auf einen Fußwurzelknochen als Druckkraft ein, driickt ihn gegen einen anderen und dieser erst wird dann zersprengt.

Ferner zeigt die hier beschriebene Gliedmaße außerdem noch besonders deutlich, dass gewisse "pathologische " Knoehen- und Gelenkumbildungen parallel "phylogenetischen " Knochen- und Gelenkumbildungen verlaufen; eine Thatsache, die für die »Ursachenforschung " von größter Wichtigkeit ist.

\section{Kap. 1. Äulseres Bild der Ver- bildung (Fig. 1)1).}

Der bei diesem Fuß an der Innenseite liegende Stiefzeh $\left(D^{\prime}\right)$ ist anffällig lang und stark, viel länger und stärker als der neben ihm liegende Stammzeh ${ }_{2}$. Ferner hat er, bereits von seinem $M-$ Körper $\left(M^{\prime}\right)$ an, eine starke Verbiegung nach außen erfahren, die zum großen Theil auf fixirter Bengestellung der Gelenke beruht, und an der besonders sein erstes und zweites Gelenk mit fixirter Beugung von fast $90 \mathrm{Grad}$ betheiligt sind. In Folge dessen zieht der Zeh mit seinen Phalangen quer über die Fußsohle Fig. 1.

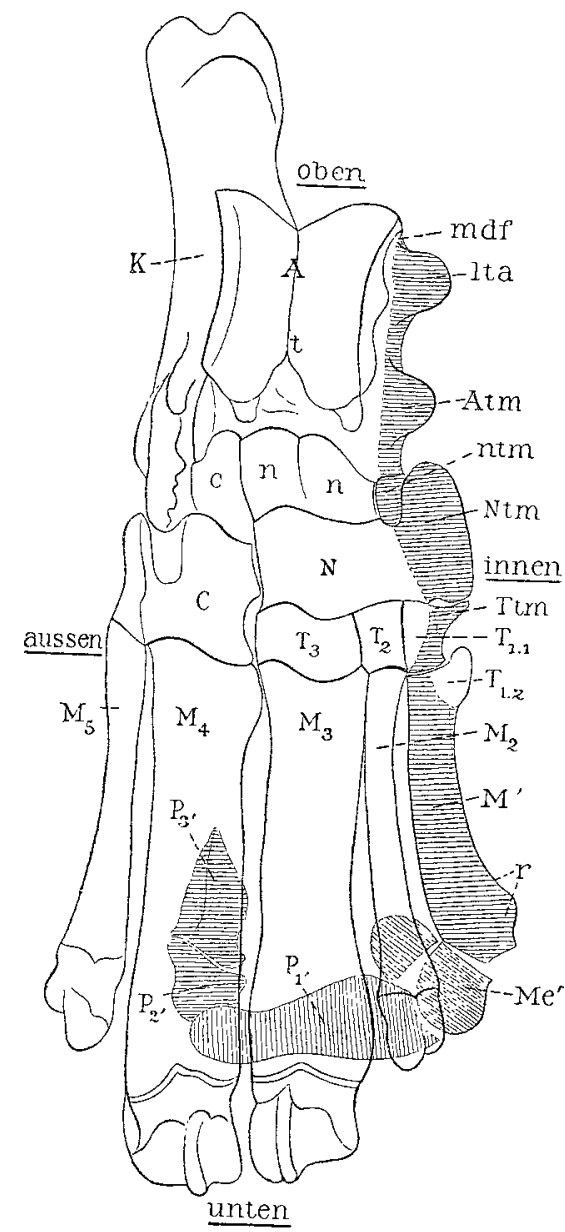
des Thieres hinweg und reicht mit seiner Spitze bis zur Mitte des Stammzeh $_{4}$. Dazu haben dann noch seine beiden letzten Phalangen eine Drehung in positiver Richtung um die Längsachse erfahren, so

1) In Fig. 1 sind die für diese Fußverbildung charakteristischen Knochenpartien durch Schraffirlinien hervorgehoben. 
dass sie mit ihrer Sohle gegen die Fußsohle des Thieres sehen, und drittens hat der Stiefzeh an seiner Innenseife über seinem $M$-Unterkopf (bei $r$ ) in der Haut einen blatunterlaufenen Fleck, der unter Einfluss starken Amniondrucks an dieser Stelle entstanden ist.

\section{Kap. II. Knochencharaktere.}

1. Astragalus (Fig. $1 \mathrm{~A}$ ).

In diesem verbildeten Fuß giebt es eine Anzahl von Knochen, welche entweder gar nicht oder so unbedentend verbildet sind, dass sie in dieser Arbeit nicht eingehend behandelt werden. Es sind vor Allem die Knochen an der Fußwurzelaußenseite und die Zehen 5 bis 2. Dagegen weicht an der Fußinnenseite bereits der Astragalus (A) sehr beträchtlich von der normalen Gestalt dadurch ab, dass zuerst seine Innenseite als Gesammtheit ein Übermaß der Entwickelung zeigt (so weit schraffirt) und dann sind an ihr auch sämmtliche Höcker

Fig. 2.

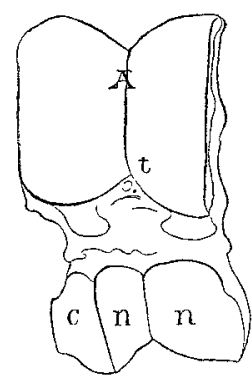
im Übermaß ausgebildet, so springt aus ihr eine Ansatzstelle fuir das Lig. tib.-ast. medial-distale $(l t a)$ als gewaltiger Höcker heraus, der an seiner Hinterseite eine querstehende Reibfläche ( $m d f)$ für die Musc. digitorum flexor medius-Endsehne trägt, während am normalen Astragalus (Fig. 2) dieser Wulst durch eine nur rauhe Knochenstelle ersetzt ist und die Reibfläche jener Sehne als flache Rinne am Knochen selbst gelegen ist. Zweitens zieht an diesem verbildeten Astragalus innen am Kopfrande eine wohlausgebildete Gelenkfläche $(n t m)$ von vorn nach hinten, die aus einer oberen, mittleren und unteren Ausbuchtung besteht und Beziehung zu einem am $N$ neu entstandenen Knochenfortsatz (Ntm) hat. Beim normalen $A$ liegt dagegen an derselben Stelle eine nur angedentete Reibfläche von gleicher, aber nicht scharf ausgeprägter Gestalt, die für das Lig. ast.-nav. mediale bestimmt ist. Drittens liegt dann an diesem $A$ unmittelbar vor dieser Gelenkfläche ein starker Knochenwulst (Atm), von dem das Lig. ast.nav. mediale an das Nav. geht und an dessen Oberseite das Lig. deltoideum wie auf einer Gelenkfläche reibt. Am normalen $A$ ist dieser Wulst dagegen gerade nur angedeutet. 


\section{Naviculare $(N)$.}

Vergleicht man ein normales Schweine- $N$ mit dem hier verbildeten $(N)$, und zwar zuerst von der Vorderseite (Fig. 3), so füllt sofort auf, dass das verbildete nicht nur die normale Gelenkfläche für den Astragaluskopf $(a)$ in wesentlich unveränderter Gestalt besitzt, sondern dass es daneben auch noch eine neugebildete Taberositas medialis (Ntm) von immenser Größe aufweist, die ungemein stark nach vorn vorspringt und an ihrer Außenseite eine Gelenkfläche (anm) trägt, welche am $A$-Kopf an der Innenseite mit der daselbst früher beschriebenen dreibuchtigen Gelenkfläche (Fig. 1 ntm) gelenkt. Am normalen $N$ ist eine derartige Tuberositas gar nicht vorhanden, ihre Stelle nimmt dort das Lig. ast.-nav. mediale ein, welches normal sehr lang, bei dem verbildeten Fuß dagegen nur wenige Millimeter lang ist und in beiden Fällen die $N$-Innenseite mit der $A$-Kopfinnenseite verbindet. Diese am verbildeten $N$ neu entstandene Tuberositas medialis (Fig 1 $N t m)$ erstreckt sich ïber die ganze Knocheninnenseite nach vorn und hinten und trägt sogar an ihrem Hinterrand eine Gelenkfläche für das $T_{1}$ (Fig. $4 t t m$ ), welche mit des Knochens normaler $T_{1}$-Gelenkfläche $\left(t_{1.1}\right)$ unter Winkelbildung zusammenstößt.

Fig. 3.

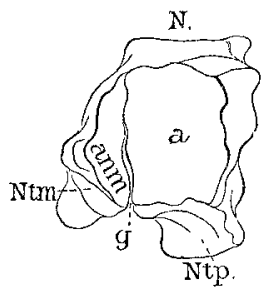

Dass selbst diesem $N$ seine Tuberositas medialis (Ntm) durchaus fremdartig gegenübersteht, wird dadurch bewiesen, dass sie ihm gegenüber sehr selbständig ist; denn obgleich sie zu ihm gehört, ist sie ihm eigentlich nur im oberen Theil fest verwachsen, während ibr ganzer unterer Abschnitt bis iiber die Mitte hinaus durch eine tiefe Grube vom $N$ getrennt ist, die man besonders gut an der $N$-Vorderseite sehen kann (Fig. $3 g$ ). Es fehlt also eigentlich nur recht wenig an der vollständigen Selbständigkeit dieser Tuberositas $(N t m)$ dem $N$ gegeniiber.

\section{Tarsale ${ }_{1}$ und Entstehn des Überzähligen.}

Bei dem vorliegenden verbildeten Fuß weicht der Knochen, der als $T_{1}$ zu gelten hat, so bedeutend von einem normalen $T_{1}$ ab, dass selbst flïchtige Betrachtung desselben erkennen lässt, dass hier der Hauptsitz dieser ganzen Fußverbildung zu suchen ist, und in der That lässt sich bis ins Detail nachweisen, dass der in diesem Fuß als $T_{1}$ auftretende Knochen nur einen Rest eines normalen $T_{1}$ darstellt, 
das, nachdem es normal angelegt war, in drei Stiicke zersprengt worden ist, in der Weise und an den Stellen, welche in Fig. $6 a$ durch Punktlinien dargestellt sind. Von seinen drei Sprengstücken (Fig. $6 a$ ) blieb beim Weiterwachśen des Fußes nur das oberste $\left(T_{1.1}\right)$ als $T_{1}$-Rest erhalten, das zweite, vorn unter ihm liegende $\left(T_{1.3}\right)$, verwuchs mit dem $N$ und bildet nunmehr am $N$ unter seiner Gleite $t_{2}$ einen abnormen, gyoßen Höcker (Fig. $4 T_{1.3}$ ), und das dritte und hinterste dieser $T_{1}$-Sprengstïcke $\left(T_{1.2}\right)$ löste aus seiner Wundfläche Superregenerativkrïfte ans und erzeugte so (Fig. $6 b$ und $c, T_{1.2}$ ) den überzähligen Zeh ( $M^{\prime}$ etc.) dieses Fußes.

Der Beweis liegt in Folgendem:

Das normale $T_{1}$ hat eine sehr charakteristische Form: es gelenkt vorn an seinem $N$ in einer Gelenkfläche (Fig. $5 t_{1}$ ), welche des $N$

Fig. 4.

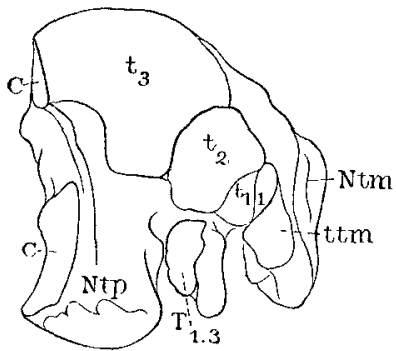

Fig. 5.

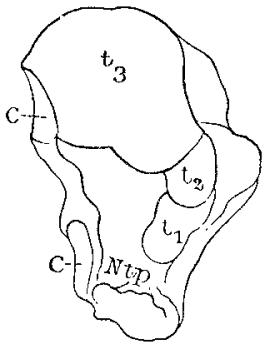

Gelenkfläche für das $T_{2}$ (Fig. $5 t_{2}$ ) von innen und unten umschlieBt, sie ist ferner sehr tief und zieht sich desshalb bis an die N-Tuberositas plantaris $(N t p)$ hinab. Ein normales $T_{1}$ gelenkt dann fermer (Fig. 6a) an seinem hinteren Ende oben mit dem $M_{2}\left(m_{2}\right)$ und unten, wo es die Form eines großen Höckers hat, ein ganz klein wenig an einem überzähligen Knochen $(S)$, der seinerseits wiederum vorwiegend an der $M_{3}$-Tuberositas plantaris gelenkt und senkrecht unter ihr steht. Am verbildeten $\mathrm{FuB}$ hat dagegen das $N$ eine nur ganz kleine Gelenkfläche für das scheinbare $T_{1}$ (Fig. $4 t_{1.1}$ ), welche nur neben der Gelenkfläche $t_{2}$ des $N$ liegt. Diese Gelenkfläche $\left\langle t_{1.1}\right)$ entspricht also auch nur dem inneren Theil der am normalen $N$ vorhandenen Gelenkfläche $t_{1}$. Unmittelbar unter ihr und genau an der Stelle, wo sonst das normale $T_{1}$ auch noch gelenkt, trägt dann aber das verbildete $N$ einen großen Höcker (Fig. $5 T_{1.3}$ ), der jedem normalen $N$ völlig fehlt und nur dadurch entstand, dass die vom verbildeten $T_{1}$ am Vorderende unten abgesprengte Partie $\left(T_{1.3}\right)$ mit $\operatorname{dem} N$ an jener Stelle verwuchs. 
Das vorliegende Stief- $T_{1}$ (Fig. $6 e T_{1.1}$ ) gelenkt dann ferner, wie ein normales, an seinem Hinterende oben mit dem $M_{2}$, dagegen ist sein hinteres Ende unten stark verkürzt, hat daselbst also auch nicht mehr die Form eines großen Höckers und reicht desshalb auch nicht mehr bis zur $M_{3}$-Tuberositas plantaris und zu dem überzähligen Knochen $(S)$ hinab, welcher am normalen Fuß an derselben Stelle liegt und mit dem $T_{1}$ gelenlst. Im Gegentheil: es schiebt am verbildeten Faß der überzählige $M^{\prime}$ einen scharf von ihm abgesetzten Fortsatz $\left(T_{1.2}\right)$ in der Art zwischen das $T_{1}$ und den überzähligen Fußknochen $(S)$, dass dieser Fortsatz $\left(T_{1.2}\right)$ oben mit dem $T_{1}\left(T_{1.1}\right)$, unten mit dem überzähligen Knochen $(S)$ gelenkt, während der iiberzählige $M^{\prime}$ selbst außerdem noch eine eigne besonders große Gelenkfläche fuir das pathologische $T_{1}$ besitzt. - Dieser überzählige $\mathbf{M}^{\prime}$-Fortsatz $\left(T_{1.2}\right)$, dem gar kein Analogon an irgend einem normalen $M$

Fig. 6 .
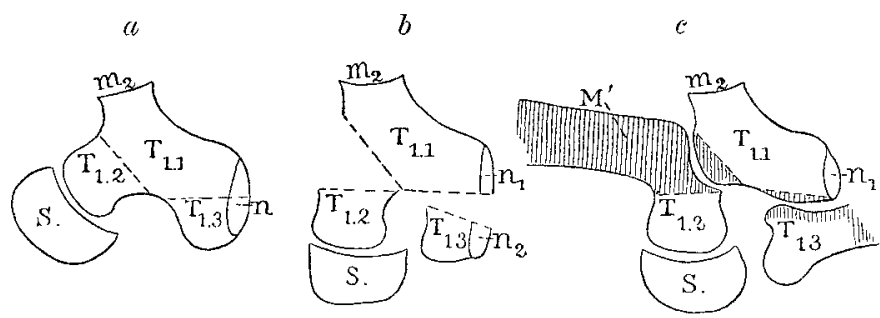

entspricht, beweist durch seine Gestalt und Lage unwiderleglich, dass er gleich ist jenem Theil eines normalen $T_{1}$ (Fig. $6 a T_{1.2}$ ), welcher als Höcker bis zum überzähligen Fußknochen (S) geht, woraus weiter folgt, dass am verbildeten Fuß vom $T_{1}$ ein entsprechendes Stiuck abgesprengt worden ist und zur Entstehung des ïberzähligen $M^{\prime}$ nebst Zeh Veranlassung gab.

Die Art, wie das geschah, war folgende (Fig. 6b): Das $T_{1}$ hatte durch das Absprengen eines hinteren Endes zwei Wundflächen erhalten, die durch Auseinanderklaffen am Wiederverwachsen verhindert wurden; sie begannen nun bald Superregenerativkräfte auszulösen, aber nur dem unteren $T_{1}$-Sprengstiick $\left(T_{1.2}\right)$ gelang ihre volle Entfaltung und zwar desshalb, weil seinem Regenerat kein Wachsthumshindernis entgegentrat, dagegen wurde dieses Regenerat selbst zum Wachsthumshindernis für das Regenerat des mittleren $T_{1}$-Sprengstiicks $\left(T_{1.1}\right)$, indem es sich im Wachsen vor dieses schob und ihm so den Raum zum Weiterwachsen nahm, sonst wäre auch - nach Analogie von Vordergliedmaßen - aus diesem $T_{1}$-Stück ein überzähliger Zeh heraus- 
gewachsen, der FuB also 6-zehig geworden. Dafur bildeten nun die beiden Regenerate, wo sie sich beriihrten, Gelenkflächen für einander aus, so dass dadurch das mittlere $T_{1}$-Bruchstiick $\left(T_{1.1}\right)$ als pathologisches $T_{1}^{\prime}$ zum ïberzähligen Zeh als Träger des $M^{\prime}$ in Beziehung trat.

\section{/ Kap. III. Grund der $\mathrm{T}_{1}$-Zersprengung.}

Die Art, wie das $T_{1}$ zersprengt wurde, war folgende: Eine drückende Kraft griff, von hinten nach vorn streichend, das $T_{1}$ am hinteren Ende unten an, iibte einen Druck auf dasselbe aus und driickte dadurch zuerst sein Vorderende mit dem unteren Abschnitt gegen den $N$-Körper. Als der Druck dann noch intensiver wurde, zersprang das $T_{1}$ unter dem Krafteinfluss streng nach technischen Principien in seine drei Theilstïcke.

Ausgelöst wurde dieser Druck auf das $T_{1}$ durch eine Amnionfalte, welche den unter der $\mathrm{Mt}_{s_{3}}$-Basis liegenden iiberzähligen Fuß-

Fig. 7.

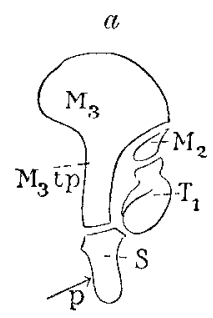

knochen $(S)$, dessen Längsachse normaler Weise senkrecht steht, in horizontale Lage umkippte, wie das Fig. 7 zeigt. Es stellt diese Figur sämmtliche an der $T_{\perp}$-Zersprengung betheiligten Faktoren dar: Den Mts $s^{-}$ Kopf $\left(M_{3}\right)$, den daneben liegenden $M t s_{2}$-Kopf $\left(M_{2}\right)$, das $T_{1}$ and endlich den vorwiegend unter dem $\mathrm{Nts}_{3}$ liegenden iiberzähligen Fußknochen

$(S)$, der am $\mathrm{Hts}_{3}\left(M_{3}\right)$ in einer horizontal liegenden Gelenkfläche gelenkt. Auf diesen uberzähligen Knochen nun, und zwar auf seine Außenseite, hat eine Amnionfalte einen starken Druck $(p)$ nach innen, oben und vorn ausgeiibt, dadurch wurde der iiberzählige Knochen aus seiner normalen Stellung so hinausgedrängt, dass er mit dem Innenrand seiner Oberfläche zwischen die $M t_{3}$-Tuberositas plantaris $\left(M_{3} t p\right)$ und das $T_{1}$ gedrängt wurde, während er gleichzeitig Horizontalstellung einzunehmen bestrebt war. Die Folge davon war, dass zuerst das $T_{1}$ aus seiner Stellung neben dem $T_{3}$ nach innen gedrängt wurde, worauf es, nachdem der Druck zu groß geworden war, in die drei Stiicke zersprang, von denen dann das hinterste untere Stiick $\left(T_{1.2}\right)$ mit dem nachdrängenden überzähligen Knochen (S) ein neues Gelenk ausbildete, während das vordere der drei $T_{1}$-Bruchstiicke (Fig. $6 T_{1.3}$ ) mit dem $N$-Körper verwuchs. 
Nachdem so aus dem $T_{1}$ zwei selbständig bleibende Theilstücke $\left(T_{1.1}\right.$ und $\left.T_{1.2}\right)$ mit je einer freiliegenden Wundfläche entstanden waren, begann an diesen beiden Wundflächen die Regeneration, deren Erfolg, wie beschrieben, in einem Fall die Kompletirung des Bruchstiicks $\left(T_{1.1}\right)$ war, im anderen das Herauswachsen eines überzähligen Zehs, während das dritte Bruchstiick des $T_{1}$ zu einem Höcker des $N$ wurde.

\section{Kap. IV. Indirekte Folgen der Amnionbeanspruchung der Fulswurzel.}

Die am vorliegenden Schweinefuß bisher beschriebenen Missbildungen können in zwei Gruppen getheilt werden: In direkte und indirekte Folgen der Amnioneinwirkung anf die Fußwurzel. Direkte Folgen jener Amnioneinwirkung sind die Verlagerung des iiberzähligen Fußwurzelknochens $(S)$ durch das Amnion and die Zersprengung des $T_{1}$ durch dasselbe in drei Stücke. Indirekte Folgen ihrer Einwirkung sind die VergröBerung der ganzen $A$-Innenseite und der daran liegenden Bandansatzstellen, ferner das Neuauftreten einer $N$-Tuberositas medialis und die Verbreiterung des $T_{1}$-Restes an der ganzen Innenseite um eine entsprechende Tuberositas medialis $(T t m)$, welche mit der $N$-Tuberositas medialis gelenkt.

Es fragt sich nun, ans welchen Gründen und auf welche Weise haben jene drei Fußknochen $\left(A, N\right.$ und $T_{1}$-Rest $)$ diese Erweiterungen erfahren?

Ein Anhänger der alten Schule der Descendenzlehre würde sofort als Grund fuir das Entstehn dieser Neubildungen angeben: Jene Knochenerweiterungen waren für den Fuß nothwendig zum Tragen des an ihm entstandenen iiberzähligen Zehs und wurden desshalb ausgebildet. An dieser Erklärnng wäre dann aber nur richtig, dass sie thatsïchlich das Tragen des iiberzähligen Zehs ermüglichen; andererseits ist es für das Thier und den Fuß aber durchaus nicht nützlich, dass der Stiefzeh an ihnen vorhauden ist und mitgeschleppt wird, denn er ist nicht nur ganz unverwendbar, sondern hängt an der Gliedmaße als todtes Gewicht und stört sogar ihre Bewegungen sehr wesentlich. Also auch hier, wie iiberall, ist der Nutzen nicht die Entstehungsursache der Neubildungen, sondern sie entstehen in diesem Falle ausschließlich in Folge direkter Einwirkung des Stiefzehs auf die Fußwurzel, und zwar entwickeln sie sich dabei durch Verknöcherung jener normalen Bandabschnitte der verbildeten Fußwurzel, auf welche der daran hängende Stiefzeh einen abnorm starken Zug ausgeälbt hat; denn Zngspannung von iibernormaler Intensität erzengt im 
Wirbelthierskelet Bandverknöcherungen, wie ich bereits in meiner Arbeit: Das Entstehen der Gelenkflächen ausführlich ans einander gesetrt habe.

Die Bänder oder Bandpartien, welche in dieser Weise am verbildeten FuB durch Zugiibermaß in Anspruch genommen wurden, lagen

Fig. 8.
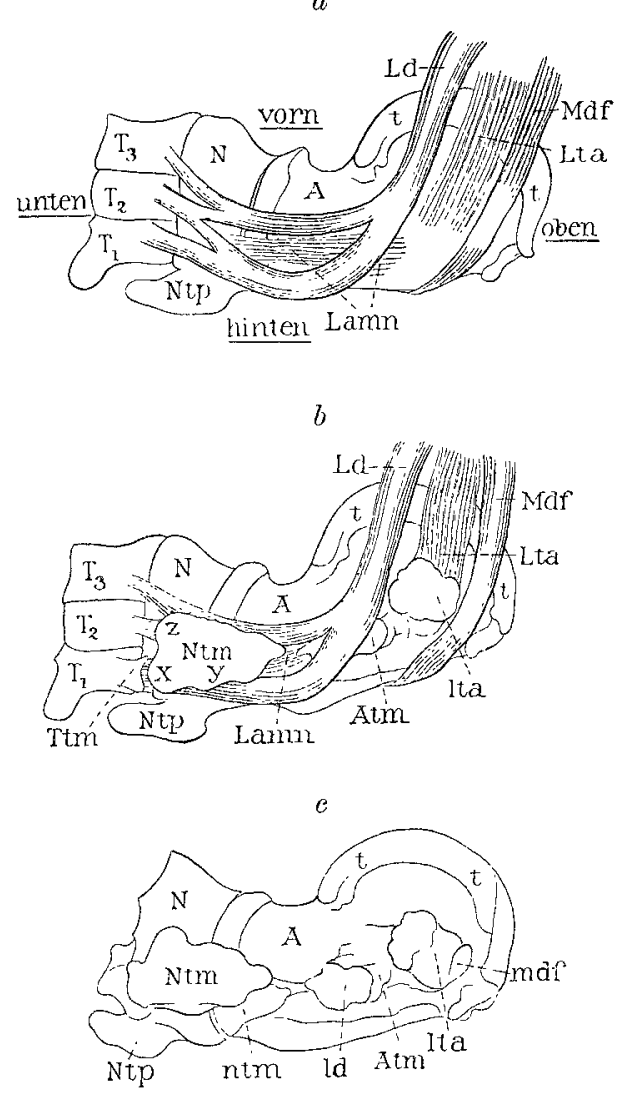
natürlich sämmtlich auf der Fußinnenseite, zogen an ihr von oben nach unten und waren folgende (Fig. $8 a$ ): Das Lig. tib.-ast. medialdistale $(L t a)$, welches verhindert, dass $\operatorname{der} A$ von der Tibia entfernt wird, dann das Lig. ast.-nav. mediale (Lamn), welches $A$ und $N$ an einander hält, dann das Lig. deltoideum $(L d)$ und zwar mit jenen Fasern, welche - zum Theil verwachsen mit dem Lig. ast.-nav. mediale - an der $N$-Innenseite inseriren und von dort weiter an die $T_{1}$-Innenseite ziehen. Die Ansatzstellen gerade dieser Bänder und Bandabschnitte vergrößerten sich dann anch am verbildeten Fuß auf Kosten der von ihnen entspringenden Bandfasern, die entsprechend weit von ihren Ansatzstellen aus verknöcherten:

So entstanden auf Kosten des Lig. deltoideum $(L d)$, dessen Faseru vom $T_{1}$ und $N$ aus zu verknöchern begannen, an der $T_{1}$ und $N$-Innenseite je ein Höcker, die $T_{1}$-Tuberositas medialis und der untere Abschnitt der $N$-Tuberositas medialis, die dann auf Bandkosten so weit gegen einander wuchsen, dass sie zum Schluss in der beschriebenen Gelenkneubildung $(n t m-t t m)$ an einander stießen. Dieser $N$-Höcker (Fig. $8 b, x)$ verwuchs aber gleichzeitig mit dem Höcker der $N$-Innenseite (Fig. $8 b, y$ ), welcher durch Verknöchern aus dem Lig. ast.-nav. mediale entstand und beide 
bilden nunmehr die am $N$ neu entstandene Tuberositas medialis (Ntm), die am $A$-Kopf an der Innenseite an der Stelle $(n t m)$ gelenkt, wo beim normalen Thier das Lig. ast.-nav. mediale eine Reibfläche hat, während die Ansatzstelle dieses Bandes am $A$-Kopf gleichfalls auf Bandkosten enorm gewachsen ist und den Höcker Atm bildet.

Gerade dieses verbildete Lig. ast.-nav. mediale zeigt übrigens bis ins Detail die Art, wie solche Bandrerknöcherungen entstehen. Denn an dem verbildeten Fuß ist von diesem Band nur ein Rest von wenig mm Länge zuriuckgeblieben, der die beiden auf Bandkosten entstandenen Knochenzapfen ( $N$ tm and $A t m$ ) verbindet, während das Übrige verknöcherte; und da bei dieser Bandumwandung die Bandumgebung wenig oder gar nicht mit verändert worden ist, sehen wir, dass die auf diese Weise entstandenen Knochenfortsätze des $N$ und $A$ genau die Stelle jener verknöcherten Bandpartien einnehmen.

Endlich ist dann am verbildeten $\mathrm{FuB}$ auch noch das Lig. ast.-tib. distal-mediale (Lta) von seiner Ansatzstelle am $A$ aus sehr stark verknöchert, wodurch der $A$ den an seiner Innenseite hinten liegenden starken Höcker $(l t a)$ erhielt. Und da währenddessen außerdem die A-Innenseite - als Gesammtarbeit von zwei Bändern - ibernormalen Zug erhielt, so war auch für sie als Gesammtheit die Anregung zur Vergrößerung gegeben, dem sie dann auch Folge geleistet hat.

Nebenbei sind diese Bandverbildungen - entstanden als indirekte Folgen der Amnioneinwirkung auf den Fuß - neue ansgezeichnete Belege für die Berechtigung meiner früher schon erwähnten Angaben, dass im Wirbelthierskelet Bandabschnitte dann verknöchern, wenn sie übernormalem Zug ausgesetzt sind.

\section{Kap. V. Die Muskeln des Stiefzehs.}

Eine zweite indirekte Folge der Amnioneinwirkung auf den FuB liegt in dem Entstehn der Muskeln für den Stiefzeh. Es kommen dabei aber nur drei derartige Bildungen in Betracht, weil der Stiefzeh an der Oberseite keine Muskelelemente besitzt. Der starke Druck, der hier, wie wir sehen werden, auf ihn eingewirkt hat, verhinderte ibre Ausbildung.

Es hat also der Stiefzeh an seiner Unterseite zuerst zwei Interossei, welche von seiner $M I^{\prime}$-Basis und vom überzähligen Fußwurzelknochen $(S)$ entspringen, unter dem überzähligen $M^{\prime}$ neben einander hinziehen und dann mit je einer Endsehne unter dem Gelenk ${ }_{1}$ an je einem Sesambein inseriren. Sie sind wahrseheinlich als Neubildungen aus den Interossei des $D_{2}$ entstanden. 
Ganz genau ist dagegen die Herkunft der dritten Muskelpartie des Stiefzehs festzustellen; sie zieht als Eudsehnenast am Stiefzeh an der ganzen Unterseite entlang und inserirt erst an seiner Phalanx ${ }_{3}$. Sie ist demnach des Zehs lange Bengesehne und entspringt desshalb auch klar und bestimmt von der Muse.-digitorum-flexor-Iongus-Endsehne und zwar unmittelbar an der Stelle, wo diese den Endsehnenast für den $D_{2}$ absendet; sie ist also eine Nenbildung aus der gemeinsamen Beugesehne des Fußes und entstand ferner an ihr nicht ans einer Wunde, sondern aus einer völlig unverletzten Stelle, denn sie entspringł völlig eingeschlossen von anderen Partien der Sehne, so dass es nummöglich war, von außen her diese Stelle der Sehne auf irgend eine Weise selbständig und allein zu verletzen.

Auf welchem Wege dann aber in jene Sehne der Antrieb zur Ausbildung dieses iiberzähligen Endabschnitts gelangt ist, ist mir nicht klar geworden.

\section{Kap. VI. Direkter Amnioneinfluss auf den Stiefzeh.}

1. Natur des Stiefzehs und seine Amnionbeziehungen.

Der Stiefzeh hatte das Bestreben zu einem $\mathrm{Zeh}_{4}$ zu werden, der dem der linken Körperseite des Thieres entsprach, d. h. er sollte ein Spiegelbild zu dem $Z_{\mathrm{eh}}$ des Fußes werden, an dem er entstanden ist. Der Nachweis hierfür ist aber nur sehwer zu führen, weil der Zeh außerdem noch sehr stark sekundär verbildet worden ist. Dass er uibrigens kein atavistisch regenerirter $D_{1}$ ist, ist sicher, weil er drei Phalangen besitzt, also eine mehr als ein jeder $D_{1}$, dass er ferner ein $D_{3}$ oder ${ }_{4}$ sein muss, wird schon aus seiner Größe klar, da er wesentlich länger und stärker als der $D_{2}$ und ${ }_{*}$ des zugehörigen FuBes ist; dass er aber gleich einem $D_{4}$ der linken Kürperseite ist, ergiebt dam zum Sehluss ganz klar seine Phalanx 3 , die mit der erwähnten genau iibereinstimmt und nur wesentlich kleiner ist.

Sobald also dieser Zeh angelegt war, stand er unter dem Zwang seiner Anlage entsprechend auszuwachsen, dazu gehörte dann auch, dass or geradlinig nach unten wuchs. Seinem Entwickelungsdrang stellte sich aber sehr bald ein Hindernis entgegen, und zwar sobald er das Amnion berihhre, das in Folge falscher Lage die Fußvorderseite wie ein Sack eng umspannte und nun dem Weiterwachsen des Stiefzehs einen so starken Widerstand entgegensetzte, dass er in der gegebenen Richtung nicht weiter wachsen konnte, sondern nur, indem er ans seiner Wachsthumsrichtung hinaus gedrängt wurde und 
nun in eine schmale Lücke zwischen Amnion und $F u ß$ hinein zu wachsen gezwungen war. Dadurch wurde er von seinem $M$-Körper beginnend (Fig. 1) quer über die Fußsohle hin verbogen, so dass jetzt nach Beendigung dieses Wachsens seine Spitze bis zur Mitte des $M_{1}$-Körpers reicht, und so eng wurde nach unten hin der Raum, in den er sich hineindrängen musste, dass seine beiden letzten Phalangen nur hineingelangen konnten, indem sie um ihre Längsache um $90^{\circ}$ positiv rotirt wurden, so dass sie nachher und nunmehr mit ihrer Sohle direkt gegen die übrige Fußsohle schauen, während sie bei einfacher Verbiegung des Zehs nach außen mit ihrer Sohle nach anßen sehen würden. Diese Verbiegung und theilweise Verdrehung des Stiefzehs war aber nicht etwa nur eine voriubergehende, die nur so lange bestand, bis sein Kampf mit dem Amnion erloschen war, sondern es wurde der Zeh in dieser Stellung für Lebenszeit fixirt, indem er unter dem Einfluss des Amnions so umgebildet warde, dass er: die Zwangslage nicht wieder verlassen konnte, und man ist demnach voll berechtigt zu sagen, diese Zehenstellung sei eine Anpassung des Stiefzels an die Zwangslage, in welche er im Kampf mit dem Amnion gerieth.

2. Anpassung des Stiefzehs an die Zwangslage.

a. Metatarsus.

Ein Beispiel ans der Technik mag das Grundprincip dieser Zehenverbildung erläutern: Wenn ein gerader Balken mit einem seiner Stirnenden in eine Mauer eingelassen wird und daun auf sein freies Stirnende von oben her eine Kraft von gehöriger Energie etwas schräg nach vorn gerichtet drückt, so drückt sie zuerst an der Angriffsstelle in den Balken ein und dann verbiegt sie den ganzen Balken, so dass seine Oberseite zur Zugseite seiner Verbiegung, seine Unterseite zur Druckseite der Verbiegung wird; seine Oberseite demnach nur auf Zug, seine Unterseite nur auf Druck beansprucht wird. War die Kraft energisch genug, so behält der Balken diese Gestalt auch nach Entfernung der Kraft für immer bei und man sagt alsdann, die Verbiegung des Balkens hat seine Elasticitätsgrenze für Verbiegung überschritten.

Ich habe nun in meiner Arbeit über das Entstehen der Gelenkflächen (Archiv für Entwickelungsmech. Bd. I. 1894/95. pag. 124 ff.) nachgewiesen, dass anch das lebende Knorpel- und Knochengewebe, wenn bei seiner Beanspruchung auf Verbiegung seine Elasticitätsgrenze iiberschritten wird, jene Formveränderung dauernd beibehält, 
welche ihm bei der Verbiegung aufgenöthigt wurde, weil es proportional einem Druck durch Atrophie verkiunt oder im Wachsen gehindert wird, während $\mathrm{es,} \mathrm{von} \mathrm{einer} \mathrm{Zugkraft} \mathrm{über} \mathrm{die} \mathrm{Elasticitäts-}$ grenze hinaus beansprucht, proportional dieser Zugenergie über das Normalmaß hinaus wächst oder proportional zu erneutem Wachsen angeregt wird.

Wir wissen dann ferner, dass ein Balkenende, dem eine allseitig drückende Kappe aufgesetzt wird, proportional dem Druck zusammengedrü ckt wird, wobei sich die Elemente des Balkenendes im Druckgebiet stark zusammensehieben; und aus dem Nachweis der Ursachen des Knochenwachsens folgt dann, dass ein Knochen unter denselben Bedingungen in gleicher Weise verbildet werden muss.

Wenn also an dem vorliegenden Fuß der Stiefzeh durch das Amnion über seine Elasticitätsgrenze hinaus verbogen und zum Theil zusammengedrïckt worden ist, so muss das in der Gestalt und Stellung seiner Knochen nachweisbar sein. Das ist in der That möglich, und zugleich ergiebt sich dabei, dass seine Verbildung nach den eben angegebenen technischen Principien stattgefunden hat, aber mit der Modifikation, dass die verbiegende Kraft des Amnions zuerst die Knochen in den Gelenken aus der Ruhelage und an einander verschob und sie dann erst verbog, während sie zugleich bei allen Knochen außerdem noch an den Angriffsstellen besondere Druckatrophie erzeugte. Die Verbildung des Zehs und seiner Knochen ist demnach eine recht komplicirte und kann in zwei Kategorien getheilt werden. In die erste Kategorie gehören alle Verbildungen dieser Knochen, welche dadurch entstanden sind, dass die Knocken von hinten und vorn vom Amnion zusammengedrüekt wurden; während die zweite Kategorie ihrer Verbildungscharaktere auf seitliche, d. h. von innen nach außen gehende Druckbelastung der Gelenkkörper und Verschiebung derselben in den Gelenken zurïckzuführen ist.

Die Art nun, wie dieser überzählige $D_{4}$ von vorn nach hinten zusammengedriickt wurde und die Wirkung dieser Beanspruchung auf die Zehenglieder sind in Fig. $9 a$ nnd $b$ dieser Arbeit dargestellt, und zwar zeigt Fig. $9 b$ Folgendes: Auf einen normalen $D_{4}$, d. h. auf ein Gebilde, zu dem der überzählige Zeh ursprünglich werden sollte, drückt von vorn und hinten das Amnion $(a a)$ als ein viel zu enger Sack in der Richtung der Pfeile $a p$, und zwar wie die Pfeillängen ergeben, auf die Vorderseite stärker als auf die Hinterseite und auf sein unteres Ende stärker als auf das obere; die Stellen gröBten Drucks sind dabei schraffirt worden. Fig. 9 a zeigt damn das Resultat dieser 
Beanspruchung des Fingers, d. h. den vorliegenden verbildeten Finger, wobei sich zeigt, dass dessen Glieder genau an den Stellen Druckschwund erlitten haben, wo sie im Embryonalleben am stärksten auf Druck beansprucht wurden, was dann auch gleichzeitig zur Fixirung der abnormen Beugestellung des Fußes führte. In Fig. 10 and 11 sind dagegen vorwiegend die Veränderungen dargestellt, welche die Glieder dieses Zehs durch einen Druck von der Innenseite und durch gleichsinnige Versehiebung der Gelenkkörper in den Gelenken erfahren haben. Ans den Fig. 9-11 lässt sich dann das Gesammtbild der Zehenverbildung sehr klar konstruiren. Es war demnach folgendes:

Der $M^{\prime}$ (Fig. 9 $\left.a\right)$ ist an vier Stellen im Kampf mit dem Amnion verbildet worden, nämlich zuerst am Körper ziemlich tief unten an seiner Vorderkante (beir), dann in seiner unteren Epiphysennaht (uen'), drittens an seiner Unterkopfinnenseite (Fig. $10 \mathrm{mi}$ ) und viertens in der Unterkopfgelenkfläche.

Der $M{ }^{\prime}$-Körper

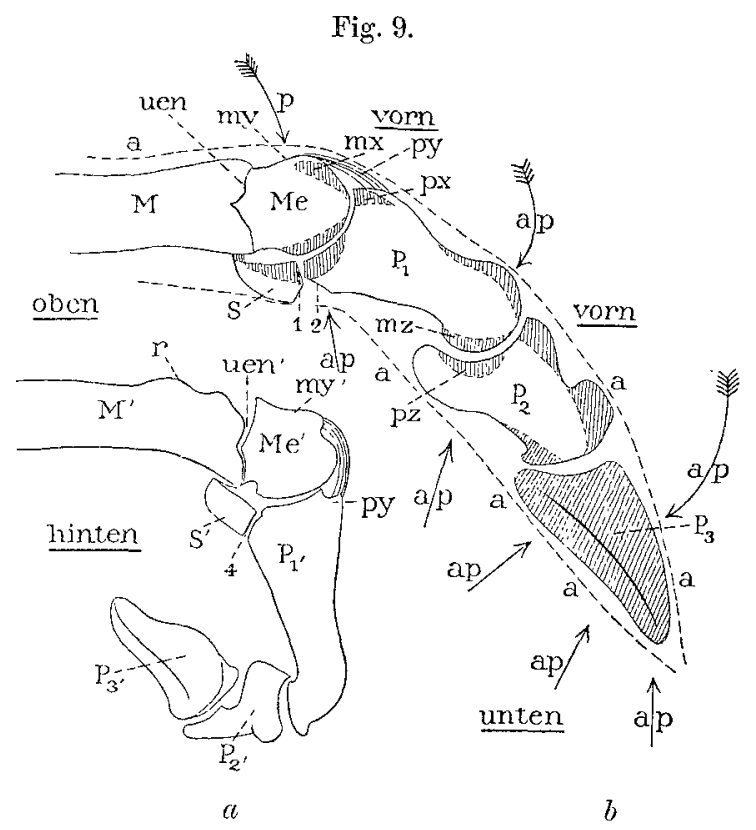
zeigt (Fig. $9 a, v$ ) in seinem unteren Drittel an der Vorderseite eine Neubildung in Form einer warzenartigen Auftreibung. Es entstand dieselbe in Folge der Reibung des Amnions auf dieser Knochenstelle, als der Zeh an ihm entlang wuchs; daneben ist dann das Knochengewebe nicht nur dieser Neubildung, sondern auch das rings um sie herum liegende, und sogar der Knochen durch seinen ganzen Querschnitt hindurch bis auf seine gegenüberliegende Außeuseite nicht von normaler Struktur, sondern schwammig verbildet, und zwar desshalb, weil das Amnion nicht nur stark an dieser Stelle rieb, sondern ihr auch sehr fest anlag und auf sie drückte; ein Beweis dafür, dass intensiver Druck auf Knochengewebe als krankmachende Ursache einwirkt. 
Durch diesen Amniondruck, welcher den $M^{\prime}$ an seiner Vorderseite von vorn her angriff, wurde außerdem der $M^{\prime}$ ind mit ilom der ganze übrige Zeh so um seine äußere Vorderkante nach hinten rotirt, dass seiue Sohle zuletzt mit der des Gesammtfußes einen rechten Winkel bildete, woranf diese Stellung beim Weiterwachsen des Thieres fixirt wurde.

Als zweite Verbildung zeigt dieser $M^{\prime}$ völlig mangelnden Schluss seiner unteren Epiphysennaht (Fig. $9 a$, zen'). Die Naht blieb offen, weil ein so starker Amniondruck von oben her den $M{ }^{\prime}$-Unterkopf traf, dass dieser vom $M^{\prime}$-Körper in jener Epiphysennaht völlig abgetrennt und nach hinten und außen rotirt wurde. Es verloren dadurch der Knochenkörper und -Kopf jeden harmonischen Zusammenhang iu der Naht und die Folge davon war ein so unregelmäßiges Wachsen dieser Knochentheile in der Naht, dass schon desshalb eine Verkuöcherung der Naht überhaupt nur schwer hätte eintreten können; vor Allem aber unterblieb sie wohl, weil der starke Amniondruck das Knochenwachsthum in der Naht bis zur Erschöpfung gehemmt hat. Die Naht persistirte desshalb für immer und der Zeh gewann dadurch zwischen $M^{\prime}$-Körper und seinem unteren Kopf eine Art Wackelgelenk, bei dessen Bethätigung seine Spitze ein wenig hin und her schlottert.

b. Unterer Metatarsuskopf $\left(M^{\prime}\right)$ und Gelenk $k_{1}\left(m^{\prime}-p_{1}{ }^{\prime}\right)$.

Der Stiefzeh sollte, wie bereits nachgewiesen ist, ein liuksseitiger $M_{4}$ werden, hatte also das Bestreben, ein entsprechendes Gelenk $m-p_{1}$ auszubilden. Ein solches hat folgende Charaktere: es ist ein Charniergelenk, d. h. ein Gelenk, in welchem, sobald intensive Berührung der Gelenkflächen eintritt, nur eine Art von Gelenkbewegung, und zwar eine Kreisbewegung um ein im $M$-Unterkopf gelegenes Centrum möglich ist, wodurch die Zehenspitze gehoben oder gesenkt wird (Fig. $9 a$ und $b$ ). Der Grund ist: die zur Ausfithrung. dieser Bewegung beim $M$ an der unteren Stirnseite vorhandene Gelenkfläche (Fig. $10 M$ ) besteht aus drei Lüng'sabschnitten. Der mittlere davon ist jene Längsgräte ( $m f$ ), welche bei inniger Beriihrung der Gelenkflächen bewirkt, dass die Gelenkbewegung nur die erwähnte zwangslänfige Hebung und Senkung wird; ich nenne diese Gräte desshalb die Fiihrung oder den Grat der Gelenkfläche. An dieselbe schließt sich rechts und links (Fig. $10 a, m a$ und $m i$ ) ein senkrecht stehender Kngelabschnitt an, auf welchem die eigentlichen Gelenkbewegungen des zugehörigen $P_{1}$ stattfinden, es sind der äußere und innere »Flügel " der Gelenkfläche. 
Das zu dieser $M$-Kopfgelenkfläche gehörige Gegenstiick, das am ersten Zehenglied die obere Stirnseite ïberzieht und in Fig. $10 \mathrm{~b}$ auf dem Kopf stehend gezeichnet wurde, als sei das Gelenk aufgeklappt worden, besteht aus genau denselben drei Abschnitten, also auch aus einer Führung $(p f)$ und zwei Flügeln $(p a$ und $p i)$, dieselben sind aber Vertiefungen und bilden daher ein richtiges Negativ zur zugehörigen Gelenkfläche des $M$-Kopfes, so dass man hier, wie in allen ähnlichen Fällen, sebr wohl von einem positiven und negativen Theil des Gelenks rieden kann.

Ferner liegen unter dem $M$-Kopf zwei Sesambeine neben einander (Fig. $10 a, S a$ und $S i$ ), die ebenfalls mit zu dem besprochenen Gelenk gehören. Das eine (Si) liegt von der $M$-Führung rechts, das andere $(S a)$ links. Jedes von ihnen hat vier Gelenkflächen, die in Kanten an einander stoßen. Die erste, fast senkrecht stehend, gelenkt mit der $M$-Führung, die zweite, oben horizontal liegend, mit jenem $M$ Gelenkflügel, unter dem sie liegt, und die beiden anderen (Fig. $10 \mathrm{a}$, $l$ und 2) mit dem $P_{1}$ unter dessen Facette, und zwar die vordere (1), wenn das $P_{1}$ am $M$-Unterkopf in starker Streckung steht, die untere (2) dann, wenn das $P_{1}$ am $M$-UnterFig. 10.

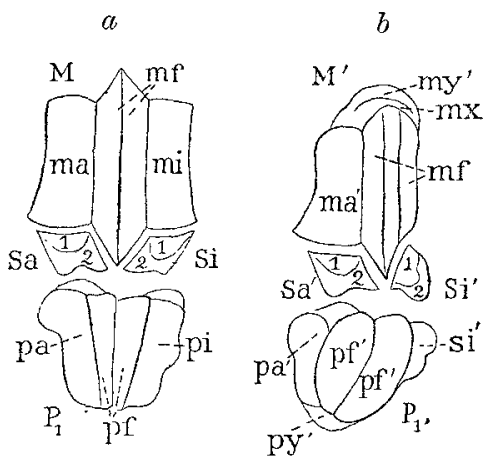
kopf in starker Beugung steht, wie das Fig. 9 in den entsprechenden Buchstaben deutlich erkennen lässt.

Am Stiefzeh ist nun das entsprechende Gelenk dadurch stark verbildet worden, dass für dasselbe einmal eine Senkung von etwa 90 Grad zur Ruhestellung wurde, gleichzeitig wurde in diesem Gelenk der Gelenkkörper $P_{1}$ durch Amniondruck nach außen verschoben und außerdem um seine äußere Oberkante nach außen rotirt.

Folgende Gestaltverïnderungen im Gelenk waren das Resultat dieser seiner dreifachen abnormen Beanspruchung:

Der starke direkte Amniondruck auf die Innenseite des $M$-Unterkopfs, des $P_{1}$-Oberkopfs und das innere Sesambein $(S i)$ hemmte (Fig. $10 a$ zeigt das Gelenk vor Beginn dieser Beanspruchung, Fig. $10 b$ den Erfolg) das Knochenwachsthum an den betreffenden Angriffspunkten so stark, dass in dem verbildeten Gelenk (Fig. 10 b) an beiden Körpern der innere Gelenkflügel ( $m i$ und $p i$ ) gar nicht zur Ausbildung kam, 
und dass auch am inneren Sesambein $\left(S i^{\prime}\right)$ die Gelenkfläche für den versehwundenen $M$-Flügel und jene Knochenpartie, auf welcher sie lag, nicht entstanden. Die Gelenkkörper im verbildeten Gelenk (Fig. $10 b, M^{\prime}$ und $P_{1}^{\prime}$ ) besitzen desshalb nur den äußeren Gelenkflügel ( $m a^{\prime}$ and $\left.p a^{\prime}\right)$ und die Führung $\left(m f^{\prime}\right.$ und $\left.p f^{\prime}\right)$ und das innere Sesambein dieses Gelenks $\left(S i^{\prime}\right)$ hat nur die Gelenkfläche für die Gelenkführung.

Dabei wurde dann noch das innere Sesambein $\left(S i^{\prime}\right)$ so stark gegen die anliegende Seite der $M^{\prime}$-Führung $(m f)$ gedrüekt, dass in dieser Führung Lage und Lauf des Sesambeins als sehwache Vertiefung mit deutlicher Grenzkante von dem übrigen Theil der Fïhrung abgegrenzt ist. -

Die Folgen der veränderten Ruhestellung des Gelenks waren folgende (Fig. $9 b$ zeigt den Beginn dieser Beanspruchung, Fig. 9a das Resultat): die auf diese Weise an beiden Gelenkflächen nie mehr mit einander in Beribrung kommenden vorderen Abschnitte ( $m x$ und $p x$ ) atrophirten durch Nichtgebrauch und ebenso die Knochenpartien, die ihnen zar Stütze dienten, dagegen verknöcherten die ïber jedem dieser Gelenkflächenabschnitte liegenden Bandansatzstellen (my und py), weil hier die Gelenkkapsel in Folge der übermäßigen Gelenkbengung im Übermaß auf Zug beansprucht wurde. Es entstand so auf jedem Knochen ein ansehnlicher Wulst ( $m y^{\prime}$ und $\left.p y^{\prime}\right)$, der beim Weiterwachsen des Knochens gleichzeitig an die Stelle des atrophirenden Gelenkflächenabschnitts hinabrückte; beide zusammen machen nunmehr eine normale Gelenkstreckung unmöglich, weil sie beim Versuch dazu schon vor Vollendung dieser Bewegung an einander stoßen und so zu Hemmfächen für das Fortschreiten dieser Bewegung werden.

Ferner driickte das $P_{1}$ mit seinem hinteren Gelenkflächenabschnitt sehr: stark gegen den hinteren Gelenkflächenabschnitt des $M$, dadurch atrophirten diese gedrückten Stellen sehr stark, wodurch einmal das Profil der $M$-Führung seine Kreisbogenform verlor und ein ganz rein nach hinten gerichtetes wurde, während sich die $P_{1}$-Führung entsprechend vertiefte. Ebenso wurde am $M$ der äußere Gelenkflügel, dureh Druck auf den hinteren Abschnitt, kleiner, der des $P_{1}$ hinten weniger gekrümmt und die Gesammtfolge davon war auch in diesem Fall die Anpassung des Gelenks an die ihm aufgezwungene Ruhestellung, d. h. Fixirung in der ihm aufgenöthigten Beugestellung.

Ferner liegen die beiden Sesambeine des verbildeten Gelenks bei der Ruhe nicht mehr normal parallel der Längsachse des Gelenks, 
sondern stehen währenddem mit ihrer Längsachse winklig am $P_{1}$, d. h. ebenfalls in fixirter Beugestellung und besitzen desshalb auch nur noch die Beugegelenkfächen (2) für das $P_{1}$ und zwar im Übermaß der Entwickelung, während ihnen die normaler Weise dariber liegende Streckgelenkfläche für das $P_{1}(1)$ völlig fehlt. -

Die drittens im Gelenk erfolgte Nachaußenverschiebung und gleichzeitige Rotation des $P_{1}$ um seine Außenkante nach außen (Fig. $11 a$ zeigt das Gelenk in normaler Ruhestellung; $11 b$ im Beginn dieser Beanspruchung, wobei die hauptsächlich druckbelasteten Gelenkpartien schraffirt sind; $11 e$ den Erfolg der Beanspruchung) bewirkte Gelenkverbildungen im Querschnitt des Gelenks, denn es wurden hierbei die beiden äußeren Gelenkflïgel $m a$ und $p a$ im Übermaß auf einander gedrickt und ihre Knochenpartien erlitten dadurch eine so starke Wachsthumshemmung, dass die beiden Gelenkflügel nunmehr mit der zugehörigen Gelenkfuihrung ( $m f$ und $p f)$ einen ganz anderen Winkel bilden, als normal ist, wie dies Fig. $11 c$ in $m a^{\prime}$

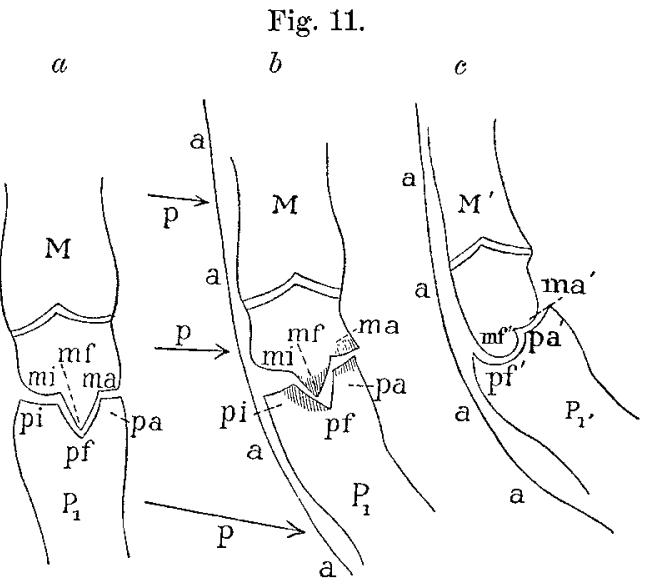
und $p a^{\prime}$ zeigt, und ferner drickte die negative Gelenkführung (Fig. $11 b, p f$ ) mit ihrer Innenseite gegen die Außenkante der positiven Gelenkführung (Fig. 11 $b, m f$ ) und die Folge davon war eine starke Abstumpfung der Kante $m f$ durch Druckschwund und eine starke Verbreiterung der Grube pf durch Ausbeulung ihres Innenrandes (wie das Fig. $11 e$ in $m f^{\prime}$ und $p f^{\prime}$ zeigt).

Weitere Veränderungen haben in diesem Gelenk nicht stattgefunden und die vorhandenen sind somit insgesammt Anpassungen des Gelenks an abnorme technisshe Beanspruchungen.

\section{c. Gelenk $k_{2}\left(p_{1}^{\prime}-p_{2}{ }^{\prime}\right)$.}

Das Gelenk ${ }_{2}$ des Stiefzehs $\left(p_{1}{ }^{\prime}-p_{2}{ }^{\prime}\right)$ ist noch stärker verbildet als sein Gelenk $k_{1}\left(m^{\prime}-p_{1}{ }^{\prime}\right)$ und zwar in drei Fällen unter Beanspruchungen, die denen analog sind, welche das Gelenk ${ }_{1}$ verbildeten, 
während eine vierte Beanspruchung nur dieses Gelenk ${ }_{2}$ angriff. Zuerst nämlich musste sich, wie schon erwähnt, der.Stiefzeh beim Wachsen von seiner Spitze an bis hierher in einen ganz engen Spalt zwischen Amnion und Fußsohle hineinklemmen und wurde daher (Fig. 9) in seiner ganzen Länge von vorn und hinten stark zusammengedrückt. Ein solcher Druck traf also auch das $P_{1}$-Unterende und zwar sehr stark und druickte es von vorn und hinten so völlig platt, dass es an Tiefe mehr als die Hälfte verlor und nun nur noch eine Art querstehender Gräte bildete (Fig. 9a). Dabei erhielt dieses Knochenunterende einen viel stärkeren Druck von vorm als von binten, was mög-

Fig. 12.

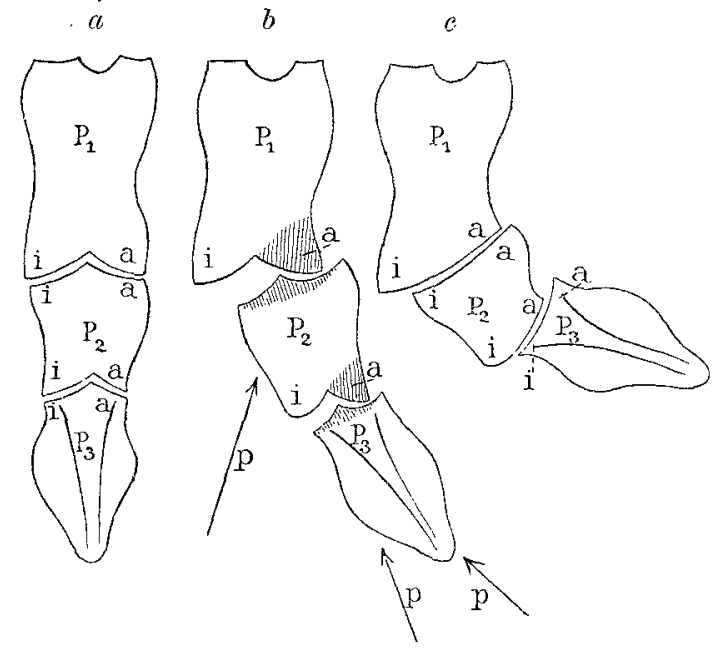

lich war, weil der wachsende Knochen dem Druck von vorn durch seine eigene Wachsthumsenergie das Gleichgewicht hielt, und in Folge dessen ist an seiner Gleite der vordere Theil ganz verloren gegangen, während der hintere Theil der Gleite an Umfang und Ausbildung kaum gelitten hat. - Dann erwarb zweitens im Gelenk $_{2}$ das $P_{2}$ eine pathologische Ruhestellung in halber Beugung (Fig. $9 a$ ), wobei es so stark auf die zugehörige $P_{1}$-Gleite drückte, dass diese dadurch zu einer solchen umgewandelt wurde, welche rein nach hinten schant.

Drittens wurde dann in diesem Gelenk (Fig. 12 zeigt den Verlanf) das $P_{2}$ am $P_{1}$ schräg nach außen verschoben, wobei es zugleich um seine Längsachse nach außen rotirte, worauf daun ein rölliges Planwerden der beiden Gelenkgleiten erfolgte, während jede von ihnen in normaler Gestalt ans zwei Flïgeln ( $i$ und $a$ ) besteht, die schräg gegen einander geneigt sind und in einer Kante zusammenstoßen, die ich als Fuihrungskante bezeichnen will. Beweis: Durch dieses Verschieben und Rotiren in dem Gelenk driiekte das $P_{2}$ mit seiner Führungskante und seinem Außenflügel so stark anf den Außenflügel des $P_{1}$, dass die beiden Drnckzonen, wie das Fig. $12 b$ andentet, in 
Folge Druckschwund anfgesaugt wurden, und die Folge davon war ein völliges Planwerden der beiden Gelenkgleiten und entsprechende Verkürzung des ganzen Zehs (Fig. 12e).

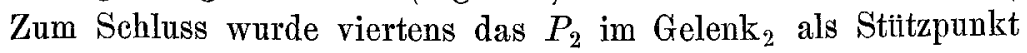
um seine Längsachse um $90^{\circ}$ rotirt, so dass nunmehr seine Sohle und die des passiv mitgeführten $P_{3}$ nach vorn, d. h. gegen die gesammte FuBsohle schauen, wobei das $P_{1}$-Hinterende als Quergräte in die zugehörige $P_{2}$-Gleite eine schrïg nach innen ansteigende Furche eindriickte.

Weitere Verbildungen zeigt dieses Gelenk nicht, also auch diese Gelenkumbildungen sind völlig aus den zugehörigen pathologischen Beanspruchungen zu erklären.

\section{d. Gelenk $k_{3}\left(p 2^{\prime}-p_{3}{ }^{\prime}\right)$.}

Auch das Gelenk ${ }_{3}$ des Stiefzehs erlitt im Kampf mit dem zu engen Amnionsack, in den es hineinzuwachsen gezwungen war, bei dreifacher pathologischer Beanspruchung Verbildungen and zwar zaerst, weil der Stiefzeh erst hier, d. h. um das Gelenk ${ }_{3}$ herum, seine stärkste Zusammendrïckung von vorn und hinten erfuhr. Die Folge davon war: Das $P_{2}$-Unterende (Fig. 9) wurde so enorm von vorn nach hinten zusammengedrückt, dass es zu einer querstehenden Gräte wurde, an welcher nur noch hinten ein Gelenkflächen-Abschnitt liegt, während gleichzeitig das $P_{3}$ so enorm an Tiefe verlor, dass seine normale Gestalt eines hohen Dreikant zu einer flachen bandartigen Knochenplatte degradirt wurde; trotzdem aber sind an dieser noch alle specifischen Charaktere der Normalgestalt nachweisbar.

Zweitens erhielt auch dieses Gelenk pathologische Ruhestellung. in schwacher Beugung und wurde dadurch am $P_{2}$ die zugehörige Gleite zu einer ganz nach hinten gerichteten, während sich das $P_{3}$ oben an seiner Hinterseite entprechend verflachte.

Drittens ist auch dieses Gelenk, indem das $P_{3}$ am $P_{2}$ nach außen verschoben und rotirt wurde, genau wie das vorige, aus einem zweiflitgeligen zu einem Plangelenk geworden, wie die Fig. 12 ergiebt.

Erwähnenswerth wäre dann noch, dass das zu diesem Gelenk gehörige Sesambein, das normal unter dem $P_{2}$-Unterende liegt und für dasselbe zwei Gelenkflächen hat', die dachförmig an einander stoßen, während es gleichzeitig mit seiner Unterseite an der $P_{3}$-Oberseite hinten gelenkt, auch hier im Wesentlichen normal erhalten ist. 


\section{Kap. VII. Direkter Amnioneinfluss auf die Stammzehen.}

Es bleiben nun noch einige Verbildungen zu besprechen, welche, wie die des Stiefzehs, zu den direkten Folgen der Amnioneinwirkung auf den Fuß gehören. Das Amnion driickte nämlich nicht nur auf den Stiefzeh und verbildete iln, sondern es beeinflusste gleichzeitig den eigentlichen $\mathrm{Zeh}_{3}$ und ${ }_{4}$ des $\mathrm{Fuhes}$, indem es auf deren Spitze einen Druck ausïbte.

Am stärksten war dabei sein Einfluss auf den $\mathrm{Zeh}_{4}$, da dieser am normalen Schweinefuß ohnehin ein wenig länger als der $Z_{e h}$ ist und daher mit seiner Spitze über ihn entsprechend hinausragt. Beide Zehen suchten nun diesem anormalen Amniondruck durch Gelenkbewegungen auszuweichen. Ihre sämmtlichen Gelenke erhielten

Fig 13.
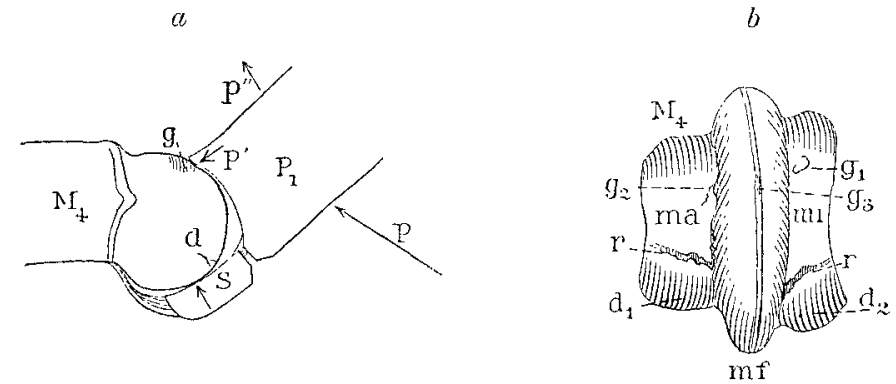

dadurch pathologische Ruhestellung in schwacher Streckstellung und damit zusammenhängende schwache Verbildung. Am stärksten war

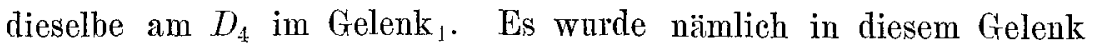
(Fig. 13 a) das $P_{1}$ in der Weise in pathologische Streckstellung gebracht, dass es vor Vollendung seiner normalen Streckbewegung mit sainem oberen Gelenkflächenrand (bei $g$ ) gegen die $M_{4}$-Kopfgelenkfläche gedrüekt wurde und nun um diesen Stiutzpunkt als Centrum einen kleinen Kreisbogen nach oben beschrieb. Dadurch driickte sich sein oberer Gelenkflächenrand mit seinen vorspringenden Flïgelpartien ін die $M_{4}$-Kopfgelenkfläche ein (Fig. $13 b, g_{1}$ und $g_{2}$ ) und gleichzeitig drückte sich der obere Rand der $P_{1}$-Führung in den gegenüberliegenden Theil der $M_{4}$-Kopfführung (Fig. $13 b, g_{3}$ ). Die Folge davon ist, dass in der $M_{4}$-Kopfgelenkfläche die Führung an der betreffenden Stelle eine Vertiefung trägt, während gleichzeitig neben derselben in jeden Gelenkflügel eine ähnliche Grube eingedrückt worden ist, die durch Schwund des Gelenkknorpels an dieser Stelle fixirt wurde. 
W:̈hrend diese Gruben $\left(g_{1}\right.$ und $\left.g_{2}\right)$ in den Knorpel eingedrückt wurden, entstand gleichzeitig an ihrer Umrandung eine so starke Zugspannung, dass dadurch rings um jede Grabe im Gelenkknorpel ein Einriss entstand, der später als Riss fixirt wurde, so dass nunmehr jede von diesen beiden Gruben durch eine feine Rinne, $d$. h. durch den nicht ganz verheilten Einriss, begrenzt wird.

In Folge dieser pathologisehen Gelenkstreckung wurden aber auch die zu diesem Gelenk gehörigen beiden Sesambeine (Fig. 13a, S), welche unter dem $M_{4}$-Kopf liegen, abnorm stark gegen den $M_{4}$-Kopf gedriickt, da sie dem $P_{\mathrm{L}}$, mit welchem sie durch Bandmasse verbunden sind, in der pathologischen Gelenkbewegung folgten, und so iiber das Normalmaß hinaus emporgehoben wurden. Auch sie erzeugten dadurch am $M_{4}$-Kopf in den Gelenkflügeln dentliche Druckstellen (Fig. 13b, $d_{1}$ and $d_{2}$ ), die von je einer, durch Zerreißen des Gelenkknorpels am Druckrande entstandenen Rime $(r)$ begrenzt werden.

Dieses Zerreißen des Gelenkknorpels am Rande einer Druckstelle ist eine pathologische Erscheinung geringsten Grades, die sehr hänfig sogar an SchweinefüBen gefunden wird, die sonst nicht weiter verbildet sind; sie ist aber auch desshall, wichtig, weil sie den ersten Anfang einer Knochenzersprengung darstellt.

\section{Kap. VIII. Schlussfolgerungen.}

1. Verlauf von Knochen- und Gelenkumbildungen.

Die Resultate dieser Untersuchungen bestïtigen, wie durch Vergleichen konstatirt werden kann, alle Angaben, die ich in friiheren Arbeiten iiber das Entstehn der Knochen- und Gelenkformen gemacht habe, denn es zeigt sich auch an diesem Objekt, dass Knochen und Knorpel im Wachsen dnreh Druck gehemmt werden, während sie durch Zug im Wachsen, bis iiber das Normalmaß hinaus, begiinstigt werden kümnen; ferner verknöcherten auch an diesem $\mathrm{FuB}$ alle Bandpartien, welche stark auf Zug bearisprucht wurden, sobald dieser Zug eine gewisse abnorme Intensität erreichte, und auch diese Fuggelenkbezirke bildeten sich unter abnormer Beanspruchung in folgender Weise um: "Es werden die Gelenkkörper auf der Beugeseite des Gelenks fester gegen eimander gedrüickt und verkürzen diese, Druckseiten ${ }^{\prime}$ proportional dem gesteigerten Druck; außerdem aber werden ihre, der Bougeseite abgewandten Seiten im Gelenk von einander entfernt, durch das verbindende Kapselband aber, sobald dieses in Zugspannung geräth, selhst in Zugspannung versetzt und verlïngern diese ,Zugseiten' proportional 
dem verstärkten Zng, wobei die Knochen außerdem noch fur immer genau die Form annebmen, welche ihnen durch die biegende Kraft der Beanspruchung, ursprünglich nur vorïbergehend oder ideell aufgenöthigt wird. - Gleichzeitig mit den Gelenkkörpern verändern sich die Gelenkflichen: Anf der Druckseite des Gelenkapparats werden sie gegen einander gepresst, hier funktionirt also ihr Gelenkknorpel und bleibt erhalten; dagegen klaffen sie an der Zugseite des Gelenkapparats oder werden durch Verschiebung blobgelegt und verlieren daselbst den Gelenkknorpel, so weit er funktionslos wird. Außerdem aber wird drittens im Gelenk der Gelenkkapselabschnitt, welcher der Muskelliontraktion antagonistisch entgegenwirkt, in stärkere Zugspannung versetzt, dadurch zur Verlängerung und Zunahme im Querschuitt angeregt, während er zugleich von beiden Gelenkkörpern ans zu verknöchern beginnt. Die auf diese Weise am Gelenk entstandenen Knochenfortsiitze modificiren nun anch ihrerseits noch die Bowegungsfühigkeit des Gelenks und zwar dadurch, dass sie in ihm die Beweglichkeit in der Richtung der Gelenkstreckung beschränken «1).

2. Haut, Muskeln und Knochen unter gemeinsamer technischer Beanspruchung.

Ls wurde bereits friher koustatirt, dass der am Stiefzeh auf der Oberseite lastende starke Amniondruck bewirkt hat, dass auf dieser Zehenseite Muskeipartien nicht ansgebildet wurden, dagegen wurde durch den Amniondruck die Haut des Stiefzehs nur dort alterirt, wo das Amnion an der $\not Y^{\prime}$-Körperinnenseite stark gerieben hat. Hier hat sich Blut in die Haut ergossen und die Hautstelle roth gefärbt. Daraus ergiebt sich das sehr interessante Schlussresultat, dass in einem Zeh - und daher wohl auch in jedem gleichgewebigen Wirbelthierorgan - durch äußere technisehe Beeinflussung das Knochenund Muskelwachsthum leichter gestört wird als das Hautwachsthum, d. h. es ist das Knochen- und Muskelgewebe zug- und druckempfindlicher als die Haut. Das ist eine sehr wichtige Thatsache, welche manche phylogenetische Skeletcharaktere erklärt. So ist z. B. die, bei den Menschenaffen den Finger- und Zehenknochen an der Palmarfläche eigenthümliche starke Abplattung auf den Druck zurückzuführen, welchen die Baumäste auf die Finger- und Zehenteller ausüben, wenn das Thier an ihnen hängt oder klettert und es ist ferner das

1) Zn vergleichen: Torsier, Das Entstehen der Gelenkformen. Archiv f. Entwickelnngsmech. Bd. I. pag. 343. 
starke Plattwerden der sämmtlichen Hand- und Fußknochen und das Verschwinden der Hand- und Fußmuskeln bei extremen Wasserbewohnern auf den Wasserdruck zurïckzuführen, der beim Schwimmen anf die ganze Gliedmaße einwirkt.

3. Dauer phylogenetischen und pathologischen Entstehns.

In dieser Arbeit, Kap. IV, wurde nachgewieesn, dass der hier besehriebene, verbildete Schweinefuß an seiner Innenseite eine Reihe von Knochencharakteren besitzt, welche entweder Bandverknöcherungen oder Vergrößerungen vorhandener Knochenpartien sind und zum Tragen des Stiefzehs dienen. Es sind die vergrößerte $A$-Innenseite und die daran befindlichen Bandansatzstellen, ferner die neu entstandene $N$-Tuberositas medialis und die $T_{1}$-Tuberositas medialis.

Es sind das nun Charaktere, welche bei anderen Thiergattungen und-Arten als konstante Normalgebilde anftreten, z. B. bei Dasypus, and sie sind auch dort, wie ich in meiner Abhandlung iber den Prähallux nachgewiesen habe, unter dem Einfluss von Zug entstanden, deu der bei diesen Thieren besonders stark funktionirende $Z_{e} h_{1}$ auf jene Bandmassen ausübte. Die Entwickelung dieser Fußcharaktere findet also bei den normalen und dem hier beschriebenen pathologischen Thier aus demselben Material und unter denselben technischen Amregungen statt und ist überhaupt eine gleichartige, wenn man -- wie es mit Recht geschieht - den Stiefzeh dieses Schweines in seiner Einwirkung auf die Fußinnenseite als physiologisch gleichwerthig dem Zeh $_{1}$ jener normalen Thiere betrachtet.

$\mathrm{Da}$ ist es nun sehr wichtig, dass von vielen Autoren angenommen wird, dass "phylogenetische " Charaktere nur in einem ungehener langen Zeitraum entstehen können, während hier dieselben Charaktere pathologisch in der Ontogenese nur eines Thieres - $d$. h. in einem. außerordentlich kurzen Zeitraum - zu gleicher Ausbildung vorschreiten und diese letzte Thatsache spricht gerade nicht sehr für die Annahme, dass »phylogenetische" Charaktere zu ihrer Ausbildung eines sehr langen Zeitraums bedürfen, sondern sic stiitzt weit mehr die andere Annahme, der ich selbst schon lange aus anderen Gründen zuneige, dass sehr hänfig phylogenetische Charaktere zu ibrer Ausbildung nur ganz kurzer Zeit beduirfen, dass dann aber die so entstandenen Thierformen eine sehr lange Periode der Formkonstanz besitzen, bis sie von Nenem zu einer neuen Umwandlung gezwungen werden. 


\section{Kap. IX. Äulsere Antriebe beim Entstehn der Hyperdaktylie.}

Es ist nicht nur in dieser Arbeit, sondern auch in früheren von mir wiederholt darauf hingewiesen worden, dass beim Entstehn von Hyperdaktylie bei Amnioten das Amnion unter Faltenbildung so stark auf die Embryonalanlage des verbildeten Fußes drückt, dass die Falte entweder direkt in die Fußanlage einschneidet, oder sie so verbiegt, dass Tarsalelemente dureh sie zersprengt werden, aus deren Wunden dann die iuberzälligen Finger oder Zehen herauswachsen. Es wurde dann ferner angegeben, dass in dem hier beschriebenen Fall - und anch in fast allen anderen ist es so - das Amnion als ein viel zu. enger Sack den weiter wachsenden Fuß auch noch nach Ausbildung des Stiefzehs so fest umschloss, dass dadurch größere Abschnitte desselben weiter verbildet oder gar zum Schwinden gebracht wurden. Es entsteht nun die Frage: Wie kann eine solche Amnionhant, welche unter normalen Verhältnissen ïnßerst zart ist und gar keine eigene Druck- und Zngspannung besitzt, so schneidende Falten bilden und einen so starken Druck ansiben, wie hier vorausgesetzt wird?

Damit sie das kann, ist nothwendig, dass Streifen oder größere Abschnitte von ihr von außen her in stairkste Zugspannung versetzt werden, denn dadurch erlangen sie die ihnen sonst fehlende Zngund Druckspannung, mit der sie dann schneidend oder drückend wirken können; wie ja auch ein Seidenfaden oder ein ganz dïnnes Grasblättchen, in starke Zugspannung versetzt und am Finger entlang geführt, gleich einem Messer in denselben einschneidet oder bei festem Anlegen an ihn selır fest gegen ihn druicken kann.

Unter den Ursachen nun, welche Streifen oder größere Abschnitte des Amnions in eine so starke Zugspannung versetzen künnen, duirfte beim Entstehn der Hyperdaktylie abnorme Erschütterung. der Frucht fast oder ganz allein maßgebend sein. Wenn nümlich ein mit jungen Embryonen trïchtiges Thier durch Hinfallen, iibermäißiges Springen, durch Schlag vor den Leib, tibertriebene Verwendung als Arbeitsthier oder auf andere gewaltsame Weise eine Erschütterung seines Leibes von abnormer Stärke erhält, so wird dadurch der von ihm getragene Embryo, welcher in seiner Amnionflüssigkeit schwimmt, entsprechend der Heftigkeit des äußeren Antriebs aus seiner Normallage im Amnionsack herausgeworfen und wird dabei alsdann entweder direkt mit einem Abschnitt seines Körpers an das Amnion anprallen, den berührten Ammionabschnitt 
in Zugspannung versetzen und dadurch eventuell einen so starken Gegendruck von ihm erhalten, dass er an der Druckstelle mehr oder weniger verbildet wird; oder, da der Embryo durch die Nabelschnur mit dem Amnion in Verbindung steht, wird er schon beim Beginn seiner Verlagermng durch Zerren am Amnionsack Zugspannung und Faltenbildung in ihm hervorrufen und an eine solche Falte kann er daranf beim Fortschreiten seiner Verlagerung mit einer Gliedmaßenanlage anstoßen und dadurch an ihr direkt oder indirekt Wunden davon tragen, welche zur Entstehang von überzähligen Fingern oder Zehen den Grund legen. Ist der Embryo aber einmal durch einen so starken Stoß im Amnionsack verlagert worden, so wird er natürlich auch nacher seine Normalstellung darin nicht wieder erlangen nnd so kann dann das Amnion nicht nur durch direkte Gegenwirkung: beim Stoß, sondern auch nachträglich noch am Embryo Verbildungen hervorrufen. So ergeben also bei dazu geeigneten Säugethierembryonen eine änßere Erschütterung als Antrieb, Verlagerung als Mittel, eine Verwundung als Grundlage Hyperdaktylie als Erfolg und nachfolgende Verbildungen als Zugabe.

Dasselbe Verhalten zeigt ïbrigens das Amnion anch beim Entstehn der Hypermelie, der Fingergablungen und beim Entstehn anderer Superregenesen am Körper der Säugethiere und sonstiger Amnioten. -

Hieraus ergiebt sich zum Schluss, wie berechtigt das Handeln der Landwirthe ist, wenn sie in Rücksicht auf gesunde Nachzucht trächtige Mutterthiere vor äußeren Angriffen jeder Art zu wahren trachten; nur wird diese Vorsicht von ihnen gewöhnlich nicht fruih genug angewandt, sondern erst dann, wenn die Thiere durch Schwellen des Leibes auch äußerlich als trïchtig erkennbar sind; dann aber ist die Zeit für das Entstehn der schwersten Verbildungen der Frucht längst schon vorbei. Der Schutz der Mutterthiere setzt daher am besten unmittelbar nach ihrer Befruchtung oder Begattung ein. 